https://doi.org/10.15407/ujpe64.1.27

A.D. ANTIA, I.B. OKON, E.B. UMOREN, C.N. ISONGUYO

Department of Physics, Faculty of Science, University of Uyo

(Nwaniba Road, Uyo, Akwa Ibom State, Nigeria; e-mail: antiacauchy@yahoo.com,

ituenokon@uniuyo.edu.ng, emmanuelumoren@uniuyo.edu.ng, ceciliaisonguyo@uniuyo.edu.ng)

\title{
RELATIVISTIC STUDY OF THE SPINLESS SALPETER EQUATION WITH A MODIFIED HYLLERAAS POTENTIAL
}

\begin{abstract}
We have solved the Spinless Salpeter Equation (SSE) with a modified Hylleraas potential within the Nikiforov-Uvarov method. The energy eigenvalues and the corresponding wave functions for this system expressed in terms of the Jacobi polynomial are obtained. With the help of an approximation scheme, the potential barrier has been evaluated. The results obtained can be applied in nuclear physics, chemical physics, atomic physics, molecular chemistry, and other related areas, for example, can be used to study the binding energy and interaction of some diatomic molecules. By adjusting some potential parameters, our potential reduces to the Rosen-Morse and Hulthen potentials. We have present also the numerical data on the energy spectra for this system.
\end{abstract}

Ke ywords: Schrödinger wave equation, modified Hylleraas potential, spinless Salpeter equation, Nikiforov-Uvarov method, potential barrier.

\section{Introduction}

Over the years, theoretical physics has been successful in inventing the equations that describe the behavior of particles in different fields of different potentials. Some of these equations are: the Schrödinger wave equation which describes a nonrelativistic particle, the Klein-Gordon equation describing a relativistic particle of zero spin, the Dirac equation for a twobody quantum field system in a relativistic covariant formalism, and others [1-11]. The spinless two-body quantum Bethe-Salpeter equation given as

$$
\begin{aligned}
& {\left[\sum_{i=1,2}\left(\sqrt{-\nabla^{2}+m_{i}^{2}}-m_{i}\right)+\right.} \\
& \left.+V(r)-E_{n l}\right] \chi(r, \theta, \varphi)=0
\end{aligned}
$$

is a standard approximation to the Bethe-Salpeter formalism, neglecting the spin degree freedom [9]. Recently, researchers have studied the Bethe-Salpeter equation for different potentials with arbitrary $l$ and $s$ waves and have obtained the exact or approximate so-

(c) A.D. ANTIA, I.B. OKON, E.B. UMOREN, C.N. ISONGUYO, 2019

ISSN 2071-0194. Ukr. J. Phys. 2019. Vol. 64, No. 1 lutions for the wave functions of various physical systems of interest. For example, Hassanabadi et al. [12] studied the SSE with hyperbolic potential via the SUSYQM formalism. Zarrinkamar et al. [13] studied the two-body Salpeter equation with exponential potential using the SUSYQM method. Ikot et al. [14] investigated the SSE with generalized Hulthen potential using also the SUSYQM approach. A special case of a multiparameter exponential-type potential is the Hylleraas potential. The modification of this potential was done in [15] and given as

$V(r)=V_{0}\left[\frac{a+e^{-\alpha r}}{e^{-\alpha r}-1}\right]$

where $V_{0}$ and $a$ are the potential parameters, and $\alpha$ is the potential range. To obtain bound-state solutions of the Klein-Gordon equation, we solve the SalpeterBethe equation with the Hylleraas potential. Various methods have be used to provide the exact or approximate solution of the Schrödinger, Klein-Gordon, and Dirac equations together with Bethe-Salpeter equation. These methods include: the asymptotic iteration method [16], supersymmetric quantum mechanics [17], shifted $\frac{1}{N}$ expansion [18], factorization method [19, 20], Nikiforov-Uvarov method [23], and others $[21,25]$. The main aim of this research 
is to obtain an approximate bound-state solution of the spinless Salpeter equation with a modified Hylleraas potential model using the Nikiforov-Uvarov method. The potential model is defined in Eq. (2), while the approximation scheme suitable to the centrifugal term is given in Eq. (25).

\section{Generalized Parametric}

\section{Nikiforov-Uvarov (NU) Method}

The NU method was presented by Nikiforov and Uvarov [23] and has been employed to solve secondorder differential equations such as the Schrödinger wave equation (SWE), Klein-Gordon equation (KGE), Dirac equation (DE), etc. The SSE can be solved by transforming it into a hypergeometric-type equation using the transformation $s=s(x)$. Its resulting equation is expressed as

$\psi^{\prime \prime}(s)+\frac{\tilde{\tau}(s)}{\sigma(s)} \psi^{\prime}(s)+\frac{\tilde{\sigma}(s)}{\sigma^{2}(s)} \psi(s)=0$,

where $\sigma(s)$ and $\tilde{\sigma}(s)$ are polynomials of at most the second degree, and $\tilde{\tau}(s)$ is the first-degree polynomial, $\psi(s)$ is a function of the hypergeometric type. The parametric generalization of the NU method is given by the generalized hypergeometric-type equation [22] and reads (4)

$\psi(s)+\frac{\left(c_{1}-c_{2} s\right)}{s\left(1-c_{3} s\right)} \psi^{\prime}(s)+$
$+\frac{1}{s^{2}\left(1-c_{3} s\right)^{2}}\left[-\xi_{1} s^{2}+\xi_{2} s-\xi_{3}\right] \psi(s)=0$.

Equation (4) is solved by comparing it with Eq. (3), and the following polynomials are obtained:

$$
\begin{aligned}
& \tilde{\tau}(s)=\left(c_{1}-c_{2} s\right), \sigma(s)=s\left(1-c_{3} s\right), \\
& \tilde{\sigma}(s)=-\xi_{1} s^{2}+\xi_{2} s-\xi_{3} .
\end{aligned}
$$

According to the NU method, the energy eigenvalue and eigenfunction, respectively, satisfy the following system of equations:

$$
\begin{aligned}
& c_{2} n-(2 n+1) c_{5}+(2 n+1)\left(\sqrt{c_{9}}+c_{3} \sqrt{c_{8}}\right)+ \\
& +n(n-1) c_{3}+c_{7}+2 c_{3} c_{8}+2 \sqrt{c_{8} c_{9}}=0, \\
& \psi(s)=N_{n l} s^{c_{12}}\left(1-c_{3} s\right)^{-c_{12}-\frac{c_{13}}{c_{3}}} \times \\
& \times P_{n}^{\left(c_{10}-1, \frac{c_{11}}{c_{3}}-c_{10}-1\right)}\left(1-2 c_{3} s\right),
\end{aligned}
$$

where $N_{n l}$ is the normalization constant, and $P_{n}$ is the Jacobi polynomial. The other parametric constants are presented in (8)

$$
\left\{\begin{array}{l}
c_{4}=\frac{1}{2}\left(1-c_{1}\right) ; \quad c_{5}=\frac{1}{2}\left(c_{2}-c_{3}\right) ; \quad c_{6}=c_{5}^{2}+\epsilon_{1} \\
c_{7}=2 c_{4} c_{5}-\epsilon_{2} ; \quad c_{8}=c_{4}^{2}+\epsilon_{3} \\
c_{9}=c_{3} c_{7}+c_{3}^{2} c_{8}+c_{6} ; \quad c_{10}=c_{1}+2 c_{4}+2 \sqrt{c_{8}} ; \\
c_{11}=c_{2}-2 c_{5}+2\left(\sqrt{c_{9}}+c_{3} \sqrt{c_{8}}\right) \\
c_{12}=c_{4}+\sqrt{c_{8}} ; \quad c_{13}=c_{5}-\left(\sqrt{c_{9}}+c_{3} \sqrt{c_{8}}\right) .
\end{array}\right.
$$

\section{Two-Body Spinless Salpeter Equation}

The SSE for two-body particles interacting through a spherically symmetric potential in the center-of-mass system $[9,10,14]$ is expressed as

$$
\begin{aligned}
& {\left[\sum_{i=1,2}\left(\sqrt{-\nabla^{2}+m_{i}^{2}}-m_{i}\right)+\right.} \\
& \left.+V(r)-E_{n l}\right] \chi(r, \theta, \varphi)=0,
\end{aligned}
$$

where

$\chi(r, \theta, \varphi)=R_{n l}(r) Y_{l m}(\theta, \varphi)$.

For heavy interacting particles and by using a suitable transformation (see Refs. $[10,11]$ ), one can recast SSE [9] as

$$
\left[\frac{d^{2}}{d r^{2}}+\frac{2 \mu}{\hbar^{2}}\left(-\frac{l(l+1)}{2 \mu r^{2}}-W_{n l}(r)+\frac{W_{n l}^{2}(r)}{2 \tilde{m}}\right)\right] R_{n l}(r)=0
$$

where

$$
\left\{\begin{array}{l}
W_{n l}(r)=V(r)-E_{n l} ; \quad \Im=\mu\left(\frac{m_{1} m_{2}}{m_{1} m_{2}-3 \mu^{2}}\right)^{1 / 3} \\
\mu=\frac{m_{1} m_{2}}{m_{1}+m_{2}} ; \quad \tilde{m}=\frac{\Im^{3}}{\mu^{2}}=\frac{m_{1} m_{2} \mu}{m_{1} m_{2}-3 \mu^{2}} .
\end{array}\right.
$$

Substituting the modified Hylleraas potential from Eq. (2) in Eq. (11) gives

$$
\begin{aligned}
& \frac{d^{2} R_{n l}}{d r^{2}}+\frac{2 \mu}{\hbar^{2}}\left(-\frac{l(l+1)}{2 \mu r^{2}}-V_{0}\left[\frac{a+e^{-\alpha r}}{e^{-\alpha r}-1}\right]+E_{n l}+\right. \\
& +\frac{1}{2 \tilde{m}}\left[\left[V_{0}\left[\frac{a+e^{-\alpha r}}{e^{-\alpha r}-1}\right]\right]^{2}+E_{n l}^{2}-\right. \\
& \left.\left.-2 E_{n l} V_{0}\left[\frac{a+e^{-\alpha r}}{e^{-\alpha r}-1}\right]\right]\right) R_{n l}=0 .
\end{aligned}
$$


To convert Eq. (13) to a hypergeometric-type equation, we use the transformation

$s=e^{-\alpha r}$.

This gives rise to the transformation in S-dimensions as

$$
\begin{aligned}
& \alpha^{2}\left[\frac{s^{2} d^{2} R_{n l}}{d s^{2}}+s \frac{d R_{n l}}{d s}\right]+\frac{2 \mu}{\hbar^{2}}\left\{-\frac{l(l+1)}{2 \mu r^{2}}-\right. \\
& -V_{0}\left[\frac{a+s}{s-1}\right]+E_{n l}+\frac{1}{2 \tilde{m}} \times \\
& \left.\times\left[\left[V_{0}\left[\frac{a+s}{s-1}\right]\right]^{2}+E_{n l}^{2}-2 E_{n l} V_{0}\left[\frac{a+s}{s-1}\right]\right]\right\} R_{n l}=0 .
\end{aligned}
$$

Since the SSE with a modified Hylleraas potential has no exact solution, we employed the relevant approximation to the centrifugal term as

$$
\frac{1}{r^{2}} \approx \frac{\alpha^{2}}{(s-1)^{2}}
$$

Substituting Eq. (16) into Eq. (15) and simplifying the latter give

$$
\begin{aligned}
& \frac{d^{2} R_{n l}}{d s^{2}}+\frac{(s-1)}{s(s-1)} \frac{d R_{n l}}{d s}+\frac{1}{s^{2}(s-1)^{2}} \times \\
& \times\left\{-\frac{2 \mu}{\hbar^{2}}\left[\frac{V_{0}}{\alpha^{2}}\left(1+\frac{E_{n l}}{\tilde{m}}\right)-\frac{E_{n l}}{\alpha^{2}}\left(1+\frac{E_{n l}}{2 \tilde{m}}\right)-\right.\right. \\
& \left.-\frac{V_{0}^{2}}{2 \tilde{m} \alpha^{2}}\right] s^{2}+\frac{2 \mu}{\hbar^{2}}\left[\frac{V_{1}}{\alpha^{2}}\left(1+\frac{E_{n l}}{\tilde{m}}\right)-\frac{V_{0} a}{\alpha^{2}}\left(1+\frac{E_{n l}}{\tilde{m}}\right)-\right. \\
& \left.-\frac{E_{n l}}{\alpha^{2}}\left(2+\frac{E_{n l}}{\tilde{m}}\right)+\frac{V_{0}^{2} a}{\tilde{m} \alpha^{2}}\right] s-\frac{2 \mu}{\hbar^{2}}\left[\frac{l(l+1)}{2 \mu}-\right. \\
& \left.\left.-\frac{\left(V_{0}+E_{n l}\right)^{2}}{2 \tilde{m} \alpha^{2}}-\frac{1}{\alpha^{2}}\left(V_{0} a+E_{n l}\right)\right]\right\} R_{n l}=0 .
\end{aligned}
$$

Equation (17) can be simplified to

$$
\begin{aligned}
& \frac{d^{2} R_{n l}}{d s^{2}}+\frac{(s-1)}{s(s-1)} \frac{d R_{n l}}{d s}+ \\
& +\frac{1}{s^{2}(s-1)^{2}}\left[-\xi_{1} s^{2}+\xi_{2} s-\xi_{3}\right] R_{n l}=0,
\end{aligned}
$$

where

$\xi_{1}=\frac{2 \mu}{\hbar^{2}}\left[\frac{V_{0}}{\alpha^{2}}\left(1+\frac{E_{n l}}{\tilde{m}}\right)-\right.$

$\left.-\frac{E_{n l}}{\alpha^{2}}\left(1+\frac{E_{n l}}{2 \tilde{m}}\right)-\frac{V_{0}^{2}}{2 \tilde{m} \alpha^{2}}\right]$

$\xi_{2}=\frac{2 \mu}{\hbar^{2}}\left[\frac{V_{1}}{\alpha^{2}}\left(1+\frac{E_{n l}}{\tilde{m}}\right)-\frac{V_{0} a}{\alpha^{2}}\left(1+\frac{E_{n l}}{\tilde{m}}\right)-\right.$

$\left.-\frac{E_{n l}}{\alpha^{2}}\left(2+\frac{E_{n l}}{\tilde{m}}\right)+\frac{V_{0}^{2} a}{\tilde{m} \alpha^{2}}\right]$,

$\xi_{3}=\frac{2 \mu}{\hbar^{2}}\left[\frac{l(l+1)}{2 \mu}-\frac{\left(V_{0}+E_{n l}\right)^{2}}{2 \tilde{m} \alpha^{2}}-\right.$

$\left.-\frac{1}{\alpha^{2}}\left(V_{0} a+E_{n l}\right)\right]$.

Comparing Eq. (18) with Eq. (4) and evaluating (8) gives the following parametric constants:

$c_{1}=c_{2}=c_{3}=1, \quad c_{4}=0, \quad c_{5}=\frac{1}{2}$,

$c_{6}=\frac{1}{4}+\frac{2 \mu}{\hbar^{2}}\left[\frac{V_{0}}{\alpha^{2}}\left(1+\frac{E_{n l}}{\tilde{m}}\right)-\right.$

$\left.-\frac{E_{n l}}{\alpha^{2}}\left(1+\frac{E_{n l}}{2 \tilde{m}}\right)-\frac{V_{0}^{2}}{2 \tilde{m} \alpha^{2}}\right]$,

$c_{7}=\frac{2 \mu}{\hbar^{2}}\left[\frac{V_{1}}{\alpha^{2}}\left(1+\frac{E_{n l}}{\tilde{m}}\right)-\frac{V_{0} a}{\alpha^{2}}\left(1+\frac{E_{n l}}{\tilde{m}}\right)-\right.$

$\left.-\frac{E_{n l}}{\alpha^{2}}\left(2+\frac{E_{n l}}{\tilde{m}}\right)+\frac{V_{0}^{2} a}{\tilde{m} \alpha^{2}}\right]$,

$c_{8}=\frac{2 \mu}{\hbar^{2}}\left[\frac{l(l+1)}{2 \mu}-\frac{\left(V_{0}+E_{n l}\right)^{2}}{2 \tilde{m} \alpha^{2}}-\right.$

$\left.-\frac{1}{\alpha^{2}}\left(V_{0} a+E_{n l}\right)\right]=Y$,

$c_{9}=\frac{1}{4}+\frac{2 \mu}{\hbar^{2}}\left[\frac{l(l+1)}{2 \mu}-\right.$

$\left.-\frac{V_{0}^{2}}{2 \tilde{m} \alpha^{2}}\left(1+a^{2}+2 a\right)\right]=X$,

$c_{10}=1+2 \sqrt{Y}, \quad c_{11}=2+2(\sqrt{X}+\sqrt{Y})$,

$c_{12}=\sqrt{Y}, \quad c_{13}=\frac{1}{2}-(\sqrt{X}+\sqrt{Y})$.

Substituting Eq. (20) into Eq. (6), we obtain $n^{2}+n+\frac{1}{2}+(2 n+1)(\sqrt{X}+\sqrt{Y})+$ $+\frac{2 \mu}{\hbar^{2}}\left[\frac{V_{1}}{\alpha^{2}}\left(1+\frac{E_{n l}}{\tilde{m}}\right)-\frac{V_{0} a}{\alpha^{2}}\left(1+\frac{E_{n l}}{\tilde{m}}\right)-\right.$ 


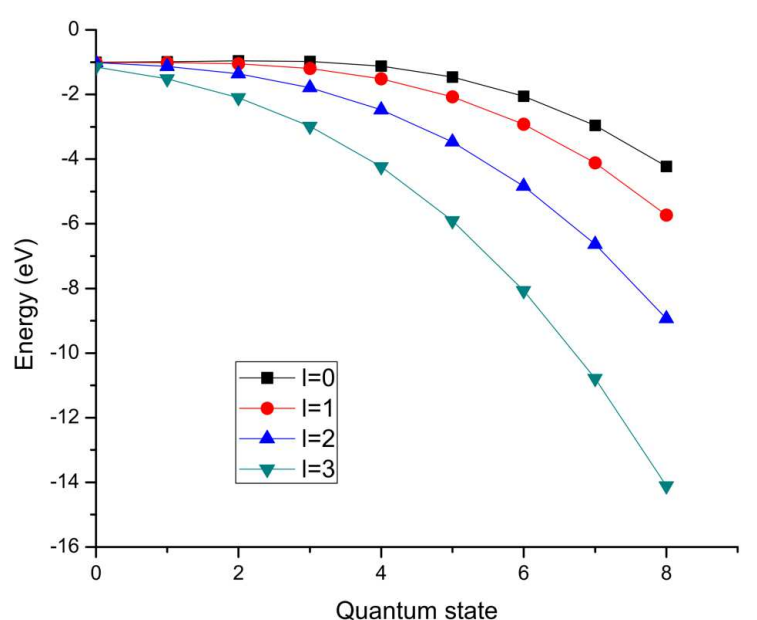

Fig. 1. Variation of the energy with the quantum state for $\alpha=0.01$

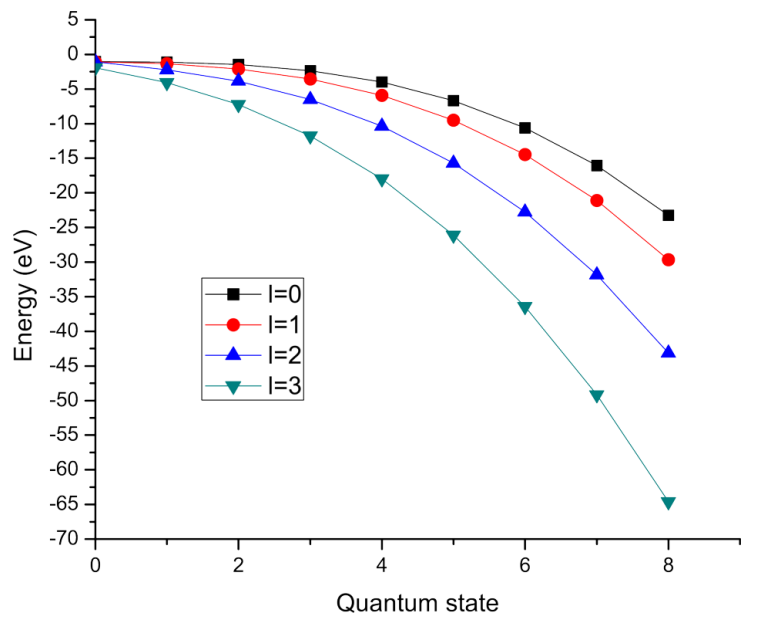

Fig. 2. Variation of the energy with the quantum state for $\alpha=0.02$

$$
\begin{aligned}
& \left.-\frac{E_{n l}}{\alpha^{2}}\left(2+\frac{E_{n l}}{\tilde{m}}\right)+\frac{V_{0}^{2} a}{\tilde{m} \alpha^{2}}\right]+ \\
& +\frac{2 \mu}{\hbar^{2}}\left[\frac{l(l+1)}{\mu}-\frac{\left(V_{0}+E_{n l}\right)^{2}}{\tilde{m} \alpha^{2}}-\right. \\
& \left.-\frac{2}{\alpha^{2}}\left(V_{0} a+E_{n l}\right)\right]+2 \sqrt{X Y}=0 .
\end{aligned}
$$

Solving Eq. (21) explicitly, we obtain the energy eigenvalues as

$$
E_{n l}=\frac{-\beta}{2 \sigma} \pm\left(\frac{\beta^{2}-4 \sigma \eta}{2 \sigma}\right)^{1 / 2}
$$

where

$$
\begin{aligned}
& \text { where }=\frac{2 \mu}{\hbar^{2} \tilde{m} \alpha^{2}}\left[\frac{2 \mu V_{0}}{\hbar^{2} \tilde{m} \alpha^{2}}(a+1)^{2}+\frac{1}{2}((2 n+1)+2 \sqrt{X})^{2}\right] \\
& \beta=\frac{4 \mu}{\hbar^{2} \tilde{m} \alpha^{2}}\left[\frac{1}{2}\left(\tilde{m}+V_{0} a\right)((2 n+1)+2 \sqrt{X})^{2}-\right. \\
& \left.-V_{0} \gamma(a+1)\right] \\
& \eta=-\frac{2 \mu}{\hbar^{2}}\left[\frac{l(l+1)}{2 \mu}-\frac{1}{\alpha^{2}}\left(V_{0} a+\frac{V_{0}^{2} a^{2}}{2 \tilde{m}}\right)\right] \times \\
& \times((2 n+1)+2 \sqrt{X})^{2}+\gamma^{2}, \\
& \gamma=n^{2}+n+\frac{1}{2}+(2 n+1) \sqrt{X}+ \\
& +\frac{2 \mu}{\hbar^{2}}\left[\frac{l(l+1)}{\mu}-\frac{V_{0}}{\alpha^{2}}-\frac{V_{0} a}{\alpha^{2}}-\frac{V_{0}^{2} a}{\tilde{m} \alpha^{2}}-\frac{V_{0}^{2} a^{2}}{\tilde{m} \alpha^{2}}\right] .
\end{aligned}
$$

Substituting Eq. (20) into Eq. (7), we have the total wave function in the form

$\psi(s)=N_{n l} s^{\sqrt{Y}}\left(1-s^{1 / 2+\sqrt{X}}\right) P_{n}^{[2 \sqrt{Y} ; 2 \sqrt{X}]}(1-2 s)$.

Equation (24) can further be reduced to

$$
\begin{aligned}
& \psi(r)=N_{n l} e^{-2 \alpha r} \sqrt{Y}\left(1-e^{-2 \alpha r} \frac{1}{2}+\sqrt{X}\right), \\
& P_{n}^{[2 \sqrt{Y} ; 2 \sqrt{X}]}\left(1-2 e^{-2 \alpha r}\right) .
\end{aligned}
$$

The total wave function expressed in a spherical coordinate system is given as

$$
\begin{aligned}
& \psi(r, \theta, \phi)=N_{n l} e^{-2 \alpha r} \sqrt{Y}\left(1-e^{-2 \alpha r} \frac{1}{2}+\sqrt{X}\right) \\
& P_{n}^{[2 \sqrt{Y} ; 2 \sqrt{X}]}\left(1-2 e^{-2 \alpha r}\right) Y_{l m}(\theta, \phi) .
\end{aligned}
$$

\section{Results and Discussions}

In order to test the accuracy of our results, we obtained the numerical values for the energy eigenvalues of the system with $\hbar=m=\mu=1, V_{1}=a=0.01$, and $V_{2}=0.02$ for $\alpha=0.01,0.02,0.03,0.04$, and 0.05 as represented in the table for various orbital angular quantum numbers $l=0,1,2$, and 3 . From the table, the results indicate negative values, which confirms to be a good necessary condition of the bound-state energy for a particle of the system. In addition, we have indicated the behavior of the energy for various quantum states for different values of the screening parameter $\alpha=0.01,0.02,0.03,0.04$, and 0.05 as shown in Figs. 1-5, respectively.

ISSN 2071-0194. Ukr. J. Phys. 2019. Vol. 64, No. 1 
Bound-State Energy with $\hbar=\mu=1, V_{1}=a=0.01, V_{2}=0.02$ for $\alpha=0.01,0.02,0.03,0.04,0.05$

\begin{tabular}{|c|c|c|c|c|c|c|}
\hline$n$ & $\ell$ & $E_{n \ell} \mathrm{eV}, \alpha=0.01$ & $E_{n \ell} \mathrm{eV}, \alpha=0.02$ & $E_{n \ell} \mathrm{eV}, \alpha=0.03$ & $E_{n \ell} \mathrm{eV}, \alpha=0.04$ & $E_{n \ell} \mathrm{eV}, \alpha=0.05$ \\
\hline \multirow[t]{4}{*}{0} & 0 & -1.00160536770 & -1.04853952979 & -1.2068669820 & -8.9930124764 & -0.62074790000 \\
\hline & 1 & -1.01266924508 & -1.06106857638 & -1.2453677502 & -1.4927152975 & -1.81012112180 \\
\hline & 2 & -1.01266924508 & -1.06106857638 & -1.2453677502 & -1.4927152975 & -1.81012112180 \\
\hline & 3 & -1.15192134466 & -1.93969205270 & -3.2620212136 & -5.1144883409 & -7.49656058007 \\
\hline \multirow[t]{4}{*}{1} & 0 & -0.98520810370 & -1.10822394550 & -1.4668391107 & -9.5604138890 & -1.55982499700 \\
\hline & 1 & -1.01146921736 & -1.35034346785 & -2.0646998331 & -3.0580823059 & -4.33574531187 \\
\hline & 2 & -1.12735173270 & -2.25027119760 & -4.1526847088 & -6.8204799555 & -10.2517095240 \\
\hline & 3 & -1.51689379440 & -4.06295984250 & -8.3346185990 & -14.318507586 & -22.0130491755 \\
\hline \multirow[t]{4}{*}{2} & 0 & -0.95554473760 & -1.45445488690 & -2.5000897895 & -11.557191533 & -4.79433396500 \\
\hline & 1 & -1.05045403580 & -2.09732274127 & -4.0080222248 & -6.6785592638 & -10.1132053829 \\
\hline & 2 & -1.36281077150 & -3.87457086910 & -8.1097175894 & -14.045638721 & -21.6793705147 \\
\hline & 3 & -2.10371783670 & -7.22804125710 & -15.812309497 & -27.835826187 & -43.2961413840 \\
\hline \multirow[t]{4}{*}{3} & 0 & -0.97877531310 & -2.33830857110 & -4.8475614161 & -15.953321525 & -11.8238925380 \\
\hline & 1 & -1.19858166814 & -3.54266939966 & -7.6216591438 & -13.329256465 & -20.6691143516 \\
\hline & 2 & -1.79098085250 & -6.49081593600 & -14.385923071 & -25.447532856 & -39.6719136620 \\
\hline & 3 & -2.98734696579 & -11.7700893900 & -26.464366045 & -47.043524803 & -73.5043965909 \\
\hline \multirow[t]{4}{*}{4} & 0 & -1.12619270290 & -4.00097322160 & -9.0493026783 & -23.709187616 & -24.1490135830 \\
\hline & 1 & -1.51905183358 & -5.92664212173 & -13.447751429 & -23.975321379 & -37.5125816817 \\
\hline & 2 & -2.47226687500 & -10.3509727160 & -23.554166908 & -42.048452865 & -65.8295044190 \\
\hline & 3 & -4.23461889731 & -17.9758274327 & -40.944588682 & -73.109377639 & -114.466429730 \\
\hline \multirow[t]{4}{*}{5} & 0 & -1.46309145220 & -6.68260963130 & -15.645462593 & -35.785173848 & -43.2702702790 \\
\hline & 1 & -2.07271098733 & -9.48940115068 & -22.027314397 & -39.579145101 & -62.1478156120 \\
\hline & 2 & -3.46685524270 & -15.7009099640 & -36.170799302 & -64.839535382 & -101.702320310 \\
\hline & 3 & -5.90911051833 & -26.1109067840 & -59.855926510 & -107.10860893 & -167.864691506 \\
\hline \multirow[t]{4}{*}{6} & 0 & -2.05168707600 & -10.6232763610 & -25.176227574 & -53.141664331 & -70.6882517300 \\
\hline & 1 & -2.91981459239 & -14.4710729777 & -33.900955614 & -61.102104150 & -96.0772077985 \\
\hline & 2 & -4.83485088700 & -22.7838685560 & -52.784925369 & -94.798170557 & -148.818423770 \\
\hline & 3 & -8.07288010849 & -36.4306021990 & -83.776070288 & -150.07033803 & -235.308721838 \\
\hline \multirow[t]{4}{*}{7} & 0 & -2.95294895900 & -16.0630325300 & -38.181798819 & -76.739043200 & -107.903552780 \\
\hline & 1 & -4.12045618935 & -21.1117699448 & -49.609105511 & -89.505129661 & -140.802348775 \\
\hline & 2 & -6.63632266500 & -31.8418040460 & -73.942061457 & -132.89491090 & -208.694856150 \\
\hline & 3 & -10.7872003824 & -49.1845931570 & -113.26900214 & -202.99860617 & -318.368361801 \\
\hline \multirow[t]{4}{*}{8} & 0 & -4.22733458610 & -23.2419474710 & -55.202384179 & -107.53769459 & -156.416770690 \\
\hline & 1 & -5.73467977054 & -29.6515976071 & -69.692107394 & -125.74893204 & -197.824430750 \\
\hline & 2 & -8.93132144810 & -43.1159824403 & -100.18578022 & -180.09659394 & -282.842662280 \\
\hline & 3 & -14.1129043020 & -64.6193245260 & -148.89073880 & -266.88285769 & -418.590327100 \\
\hline
\end{tabular}

By appropriately choosing the parameters of the modified Hylleraas potential, some potentials well known in the literature can be obtained. A few of such potentials are:

\subsection{Rosen-Morse potential}

By setting $\alpha=1$ and mapping $\alpha \rightarrow 2 \alpha$, relation (2) reduces from a modified Hylleraas potential to the 


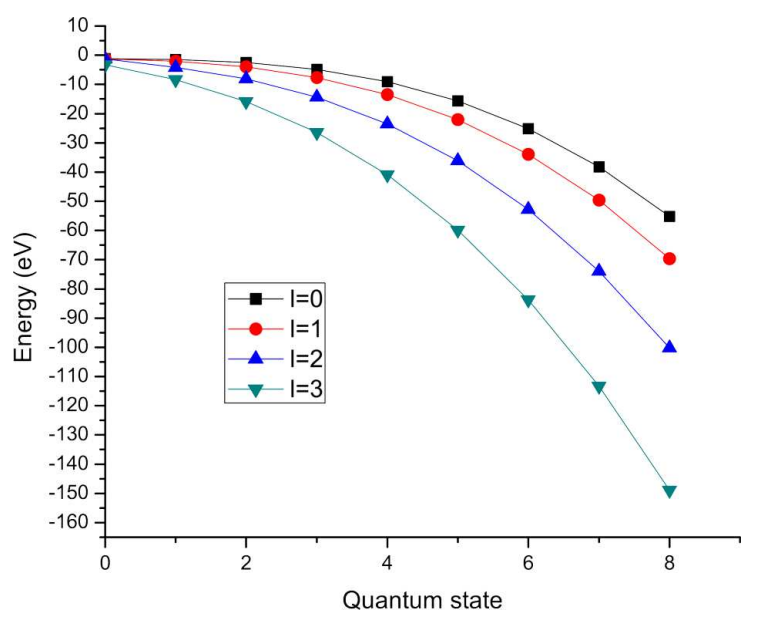

Fig. 3. Variation of the energy with the quantum state for $\alpha=0.03$

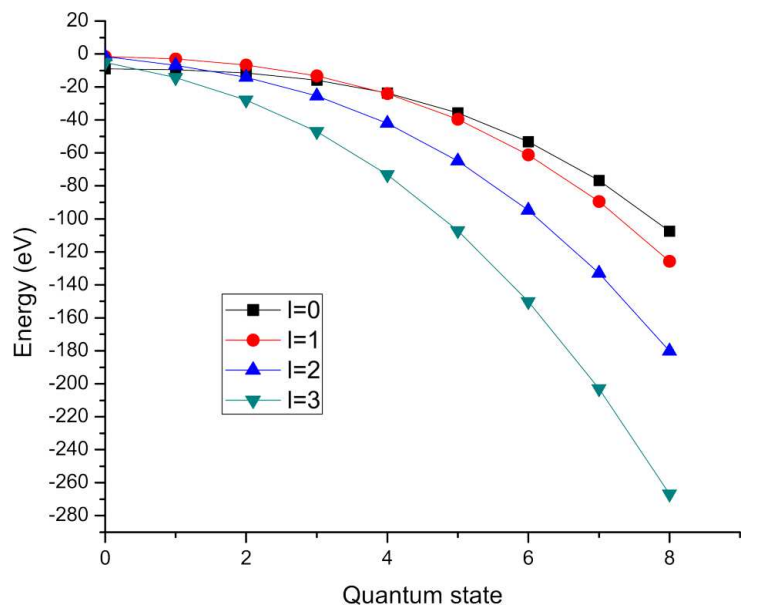

Fig. 4. Variation of the energy with the quantum state for $\alpha=0.04$

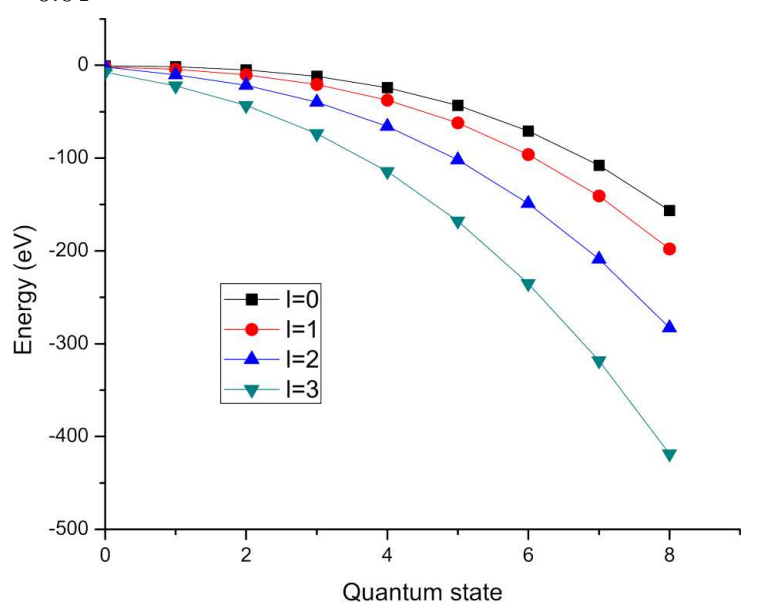

Fig. 5. Variation of the energy with the quantum state for $\alpha=0.05$

32
Rosen-Morse potential [26]

$V(r)=-\frac{V_{0}\left(1+e^{-\alpha r}\right)}{\left(1-e^{-\alpha r}\right)}$,

and the corresponding energy is obtained by substituting the above parameter into relation (22).

\subsection{Hulthen Potential}

By setting $\alpha=0$ and mapping $\alpha \rightarrow 2 \alpha$, relation (2) reduces from a modified Hylleraas potential to the Hulthen potential [27]

$V(r)=-\frac{V_{0} e^{-\alpha r}}{\left(1-e^{-\alpha r}\right)}$,

and the corresponding energy is obtained by substituting the above parameters into relation (22).

\section{Conclusion}

In this paper, the approximate bound-state solutions of the SSE with a modified Hylleraas potential with the use of the parametric Nikiforov-Uvarov method have been obtained with the help of an approximation scheme to evaluate the centrifugal term. The energy eigenvalues and the corresponding total nonnormalized wave function expressed in terms of Jacobi polynomials were obtained as well. The results of this research can be used in the study of binding and interaction energies, in the perturbation theory of neutron-proton pairs, etc. [28]. By the appropriate choice of parameters, the modified Hylleraas potential reduces to some well-known potentials such as the Rosen-Morse and Hulthen potentials.

1. E.M. Zayed, S.A. Ibrahim. Exact solutions of nuclear evolution equations in mathematical physics using the modified simple equation method. Chin. Phys. Lett.29, 6 (2009).

2. H. Hassanabadi, B.H. Yazarloo, S. Zarrinkamar, A.A. Rajabi. Duffin-Kemmer-Petiau equation under a scalar Coulomb interaction. Phys. Rev. C 84, 064003 (2011).

3. W. Lucha, F.F. Schoberl. Semi-relativistic treatment of bound state. Int. J. Mod. Phys. A 17, 2333 (2002).

4. B.I. Ita, A.I. Ikeuba. Solutions to the Schrödinger equation with inversely quadratic Yukawa plus inversely quadratic Hellmann potential using Nikiforov-Uvarov method. J. At. and Mol. Phys. 20, 1 (2013).

5. P. Maris, C.D.Robert. Dyson-Schwinger equations: A tool for hadron physics. Int. J. Mod. Phys. E 12, 197 (2003).

6. P. Maris, C.D. Robert. $\pi^{-}$and K-meson Bethe-Salpeter amplitudes. Phys. Rev. C 56, 3369 (1997).

ISSN 2071-0194. Ukr. J. Phys. 2019. Vol. 64, No. 1 
7. R. Hall, W. Lucha, F.F. Schoberl. Discrete spectra of semirelativistic Hamiltonians. Int. J. Mod. Phys. A. 18, 2657 (2003).

8. R. Hall, W.Lucha. Schrödinger secant lower bounds to semi-relativistic eigen values. Int. J. Mod Phys. A.22, 1899 (1994).

9. G.C. Wick. Properties of Bethe-Salpeter wave functions. Phys. Rev. 96, 1124 (1954).

10. E.E. Salpeter, H.A. Bethe. A relativistic equation for bound-state problems. Phys. Rev. 84, 1232 (1951).

11. S. Hassanabadi, M. Ghominejad, S. Zarrinkamar, H. Hassanabadi. The Yukawa potential in semirelativistic formulation via supersymmetry quantum mechanics approach. Chin. Phys. B 22, 060303 (2013).

12. H. Hassanabadi, S. Zarrinkamar, B. H. Yazarloo.Spectrum of hyperbolic potential via SUSYQM within the semirelativistic formalism. Chin. J. Phys. 50, 783 (2012).

13. S. Zarrinkamar, A.A. Rajabi, H. Hassanabadi. Solution of the two-body Salpeter equation under an exponential potential for any $l$ state. Few-Body Systems 52, 165 (2012).

14. A.N. Ikot, C.N. Isonguyo, Y.E. Chad-Umoren, H. Hassanabadi. Solution of spinless Salpeter equation with generalised Hulthen potential using SUSYQM. Acta Phys. Polonica A 127, 674 (2015).

15. A.D.Antia, A.N. Ikot, I.O. Akpan, O.A. Awoga. Approximate solution of the Klein-Gordon equation with unequal scalar and vector modified Hylleraas potential. Ind. J. Phys. 87, 155 (2013)

16. F. Yasuk, M.K. Bahar. Approximate solution of Dirac equation with position-dependent mass for the Hulthen potential by the asymptotic iteration method. Phys. Scr. $\mathbf{8 5}$, 045004 (2012).

17. H. Hassanabadi, E. Maghsodi, S. Zarrinkamar. Relativistic symmetries of Dirac equation and the Tietz potential. Euro. Phys. J. Plus 127, 31 (2012).

18. O. Mustapha, R. Sever. Shifted $\frac{1}{N}$ for the Klein-Gordon equation with vector and scalar potentials. Phys. Rev. A 44, 4142 (1991)

19. A.D. Antia, E.E. Ituen, H.P. Obong, C.N. Isonguyo. Analytical solution of the modified Coulomb potential using the factorisation method. Int. J. Rec. Adv. Phys. 4, 55 (2015).

20. S.M. Ikhdair, R. Sever. On solutions of the Schrödinger equation for some molecular potentials: wave function ansatz. Cent. Eur. J. Phys. 6, 697 (2008).
21. H. Hassanabadi, B.H. Yazarloo, S. Zarrinkamar, A.A. Rajabi. Duffin-Kemmer-Petiau equation under scalar Coulomb interaction. Phys. Rev. C 84, 064003 (2011).

22. C. Tezan, R. Sever. A general approach for the exact solution of the Schrödinger equation. Int. J. Theor. Phys. 48, 337 (2009).

23. A.F. Nikiforov, V.B. Uvarov. Special Functions of Mathematical Physics (Birkhäuser, 1998).

24. R.L. Greene, C. Aldrich. Variational wave functions for a screened Coulomb potential. Phys. Rev. A 14, 2363 (1976).

25. A.N. Ikot. Solution to the Klein-Gordon equation with equal scalar and vector modified Hylleraas plus exponential Rosen-Morse potential. Chin. Phys. Lett. 29, 060307 (2012).

26. S. Debnath, B. Biswas. Analytical solution of the KleinGordon equation for Rosen-Morse potential via asymptotic iteration method. EJTP 26, 191 (2012).

27. A.N. Ikot, L.E. Akpabio, E.J. Uwah. Bound state solution of the Klein-Gordon equation with Hulthen potential. Elect. J. Theor. 8, 225 (2011).

28. K.M. Khanna, G.F. Kanyeki, S.K. Rotich, P.K. Torongey, S.E. Ameka. Anharmonic perturation of neutron-proton pairs by the unpaired neutron in heavy finite nuclei Ind. J. Pure and Appl. Phys. 48, 7 (2010).

Received 02.10.18

А.Д. Антіа, І.Б. Окон, Є.Б. Уморен, К.М. Ізонгуо

РЕЛЯТИВІСТСЬКИЙ РОЗГЛЯД

БЕЗСПІНОВОГО РІВНЯННЯ СОЛПІТЕРА

З МОДИФІКОВАНИМ ПОТЕНЦАЛОМ ХІЛЛЕРААСА

$\mathrm{P}$ е $з$ ю м е

Методом Никифорова-Уварова вирішено безспінове рівняння Солпітера $з$ модифікованим потенціалом Хіллерааса. Розраховані власні значення енергії, і хвильові функції системи виражені через поліноми Якобі. Використовуючи схему наближення, оцінено потенціальний бар'єр. Результати можуть бути застосовані в ядерній фізиці, хімічній фізиці, молекулярній хімії та суміжних областях, наприклад, при вивченні енергії зв'язку і взаємодії деяких двоатомних молекул. Зміною параметрів наш потенціал можна звести до потенціалів Розена-Морзе і Хюльтена. Наведено чисельні дані про енергетичні спектри системи. 